\title{
Reviewing the quality, health benefit and value for money of chemotherapy and targeted therapy for metastatic breast cancer
}

\author{
Xavier Ghislain Léon Victor Pouwels ${ }^{1}$ (I) Bram L. T. Ramaekers ${ }^{1} \cdot$ \\ Manuela A. Joore ${ }^{1}$
}

Received: 1 June 2017 / Accepted: 30 June 2017/Published online: 8 July 2017

(c) The Author(s) 2017. This article is an open access publication

\begin{abstract}
Purpose To provide an overview of model characteristics and outcomes of model-based economic evaluations concerning chemotherapy and targeted therapy (TT) for metastatic breast cancer (MBC); to assess the quality of the studies; to analyse the association between model characteristics and study quality and outcomes.

Methods PubMED and NHS EED were systematically searched. Inclusion criteria were as follows: English or Dutch language, model-based economic evaluation, chemotherapy or TT as intervention, population diagnosed with MBC, published between 2000 and 2014, reporting life years (LY) or quality-adjusted life-year (QALY) and an incremental cost-effectiveness ratio. General characteristics, model characteristics and outcomes of the studies were extracted. Quality of the studies was assessed through a checklist.
\end{abstract}

Electronic supplementary material The online version of this article (doi:10.1007/s10549-017-4374-6) contains supplementary material, which is available to authorized users.

Xavier Ghislain Léon Victor Pouwels

xavier.pouwels@mumc.nl

Bram L. T. Ramaekers

bram.ramaekers@mumc.nl

Manuela A. Joore

m.joore@mumc.nl

1 Department of Clinical Epidemiology and Medical Technology Assessment (KEMTA), Care and Public Health Research Institute (CAPHRI) of the Faculty of Health, Medicine and Life Sciences of Maastricht University (FHML), Maastricht University Medical Centre, P.O. Box 5800, 6202 AZ Maastricht, The Netherlands
Results 24 studies were included, considering 50 comparisons (20 concerning chemotherapy and 30 TT). Seven comparisons were represented in multiple studies. A health state-transition model including the following health states: stable/progression-free disease, progression and death was used in 18 studies. Studies fulfilled on average 14 out of the 26 items of the quality checklist, mostly due to a lack of transparency in reporting. Thirty-one per cent of the incremental net monetary benefit was positive. TT led to higher iQALY gained, and industry-sponsored studies reported more favourable cost-effectiveness outcomes.

Conclusions The development of a disease-specific reference model would improve the transparency and quality of model-based cost-effectiveness assessments for MBC treatments. Incremental health benefits increased over time, but were outweighed by the increased treatment costs. Consequently, increased health benefits led to lower value for money.

Keywords Breast neoplasms · Neoplasm metastasis · Models · Economic · Cost-benefit analysis · Qualityadjusted life-years $\cdot$ Review

\section{Introduction}

Worldwide, breast cancer is the most incident and prevalent cancer among women (data from 2012) [1]. Due to the incurable character of metastatic breast cancer (MBC) and the intensive health care resource use associated with its management, MBC treatment incurs a high burden on health care budgets [2]. Policy makers therefore resort to economic evaluations to take coverage decisions concerning MBC treatments [3]. These economic evaluations are often based on decision-analytic models (or cost- 
effectiveness models) because different sources of evidence need to be synthesised and extrapolation of trial results is required to estimate the (lifetime) costs and the impact on survival and quality of life of MBC treatments. Health benefits obtained from MBC treatments are then weighted against their costs, which provide a measure of value for money used in MBC treatments.

Throughout the years, cost-effectiveness models have increasingly been used to support reimbursement decision for new (MBC) treatments and guidelines on good modelling practices have been developed [4, 5]. However, differences in model structure and assumptions, which might influence the cost-effectiveness outcomes [6], still exist between cost-effectiveness models for MBC treatments [7-10]. Study sponsorship and quality have also been reported to influence the results of cost-effectiveness assessments. Industry-sponsorship was associated with more beneficial cost-effectiveness outcomes for the treatments of interest, while higher study quality was associated with less favourable cost-effectiveness outcomes [11]. Previous research also found that the quality of the costeffectiveness assessments concerning oncology treatments has not increased over time [12]. More specifically, a previous review concerning cost-effectiveness models for MBC treatments highlighted the need for high-quality studies [13].

Because model design influences cost-effectiveness results, researchers and the European network for health technology assessment (Eunethta) have argued for increased consistency between cost-effectiveness assessments [14-17]. Eunethta consequently encourages adherence to the HTA Core model ${ }^{\circledR}$ [18] and researchers have argued for the development of disease-specific reference models; a unique model which would be used for all economic evaluations in a specific disease area $[19,20]$.

A previous review of cost-effectiveness assessments evaluating chemotherapy and TT for MBC treatment has focussed on identifying the most influential characteristics of the included economic evaluations on the cost-effectiveness outcomes [13]. However, this previous review did not only include model-based economic evaluations, did not provide an overview of model characteristics, did not assess the quality of the included studies through a standardised checklist and did not attempt to illustrate the influence of different model characteristics on study quality and outcomes. The current study consequently aims at (1) providing an overview of model characteristics and outcomes of model-based economic evaluations of chemotherapy and TT for MBC treatment, (2) assessing the quality of the included studies and (3) investigating the association between model characteristics and study quality and outcomes.

\section{Methods}

\section{Literature search and study selection}

PubMed and the National Health Services Economic Evaluation Database (NHS EED) were searched through September and October 2014 (22-10-2014). Existing reviews [13, 21-24] informed the PubMed search query which followed the PICO methodology (patient, intervention, comparator, outcome) (Online Resource, Appendix 1). The NHS EED search query was composed of the following terms: "Breast cancer" OR "Breast neoplasm". Inclusion criteria were:

- The study population includes patients diagnosed with advanced or MBC.

- The study is a model-based economic evaluation.

- Chemotherapy or TT is included as a comparator.

- The study reports an incremental cost-effectiveness ratio (ICER) with life years (LYs) and/or qualityadjusted life years (QALYs) as measure of effect.

- The study has been published in English or Dutch as a journal article between January 2000 and October 2014.

XP performed abstract screening. During full text screening, XP reviewed all studies, while BR and MJ each reviewed half of the studies. Disagreements about inclusion were resolved through discussions among all authors. XP performed reference tracking in order to retrieve potentially relevant studies. Inclusion of studies without abstract was assessed during full-text screening.

\section{Extraction of general information, model characteristics and outcomes}

XP retrieved general information on authors, country, year of publication, comparators, perspective and sponsorship of each study. Through a standardised extraction sheet, the authors retrieved the model characteristics: type of model (the health state-transition model category was composed of "Markov" state-transition models and partitioned survival models), health states, treatment effect modelling (constant or time-dependent), time horizon, extrapolation beyond trial time horizon, cycle time, adverse events (AEs) (AEs were considered as included when either costs or the effects on quality of life of AEs were incorporated in the model) and subgroup analyses included in the economic evaluations. This was performed in duplicates and discrepancies were discussed among all authors. XP also registered which treatment lines were under investigation in each study. When the treatment line was not clearly stated in the text, it was labelled as 'unclear/mix' because studies might investigate a treatment which is administrated in different treatment lines. 
XP extracted information on model inputs: utilities, utility elicitations methods, the type of AEs included and the population (hormonal and human epidermal growth factor receptor 2 (HER-2)-statuses). The following study outcomes were extracted: total LY, QALY and costs for each comparator, incremental costs and effects (incremental LY (iLY) and/or incremental QALY (iQALY)) and ICERs. Total costs, incremental costs and ICERs were converted to the year 2013 by using the Consumer Price Index of each country [25-27]. Costs were adjusted to US\$ 2013 and then to $€ 2013$ by using the Purchase Power Parity [28]. ICERs were rounded to the nearest thousand (or hundred if smaller than 1000). The Net Monetary Benefit (NMB) of each comparator and the incremental NMB (iNMB) of each comparison at a willingness-to-pay threshold of $€ 40,000$ per QALY were calculated.

\section{Quality assessment}

Quality assessment of the studies was performed based on a previously used checklist [23] which consisted of the CHEC checklist [29] and additional items suggested by Soto [30]. These additional items concern the type of model, the description of the model and the source of data used in the model. Quality indicators were scored as follows: yes/complete details given in text (1); no/no details given (0); not clearly stated within text, references given (N.C.) and not applicable (N.A.) [23]. Two authors assessed each study (XP and BR or MJ). Disagreements were resolved through discussions among all authors. The number of items rated as 'yes/complete details given' were summed up for each study in order to obtain an indication of study quality. The checklist contained 26 items.

\section{Association of model characteristics with study quality and outcomes}

Graphic plots were used to investigate the association between study quality and study sponsorship, publication year, iQALY and iNMB. Study quality was represented in percentage of correctly described items ('yes/complete details given in text') from the quality checklist. Furthermore, the association between study outcomes (iNMB and iQALY) and publication year as well as time horizon was explored. A lifetime time horizon was defined as 20 years, as this approximates lifetime in this condition. Finally, the association between iQALY and iNMB was investigated.

\section{Results}

\section{Literature search}

The literature search provided 1167 records. From those, 208 were duplicates, 19 were excluded based on language restrictions, 1 was excluded based on its publication date and the abstracts of 9 studies were not available. This resulted in 930 records eligible for abstract screening; of those, 863 were excluded. Full-text screening was performed on 77 articles (67 studies identified through abstract screening, 9 studies without abstract and 1 potential relevant study identified through reference tracking [31]). Twenty-four studies [8, 9, 32-53] were included (Fig. 1).

\section{General information and models' characteristics of the studies}

Studies were performed in Europe $(N=14)$, North America $(N=9)$ and South America $(N=1)$. Funding by a pharmaceutical company was reported by 11 studies. Two studies used only LY as outcome for the cost-effectiveness assessment, 10 used only QALY and 12 used both LY and QALY. Three studies used a societal perspective, twenty a health care/payer perspective and one used both societal and health care perspectives. The populations in the studies differed with respect to hormonal status and HER-2 status. Studies investigated interventions in different treatment lines (Table 1). The 24 studies provided 50 comparisons of treatments: 20 concerned chemotherapy and 30 concerned TT. Seven specific treatment comparisons were represented in multiple studies, totaling 20 comparisons, six of them being the same comparisons presented from two different perspectives (health care and societal). The remaining comparisons were only reported in one of the included studies.

Most studies used a health state-transition model $(N=18)$. The remaining studies used a decision-tree $(N=2)$, a combination of decision-tree and health statetransition model $(N=1)$ or did not clearly report which type of model was used $(N=3)$. Most (18 out of 19) studies using a health state-transition model (either combined with a decision-tree or not) included at least the following three health states: stable/progression-free disease, progression and death. Six of these studies also incorporated a response health state. All studies included AEs, but the number and types of AE differed (Online Resource, Appendix 2). Two studies stated they included AEs but did not provide details on which (and how) AEs were incorporated in the model $[51,53]$. Nine studies used a lifetime time horizon, nine studies used a fixed time horizon (varying between 1 and 12 years) and six studies did not clearly define or report their 


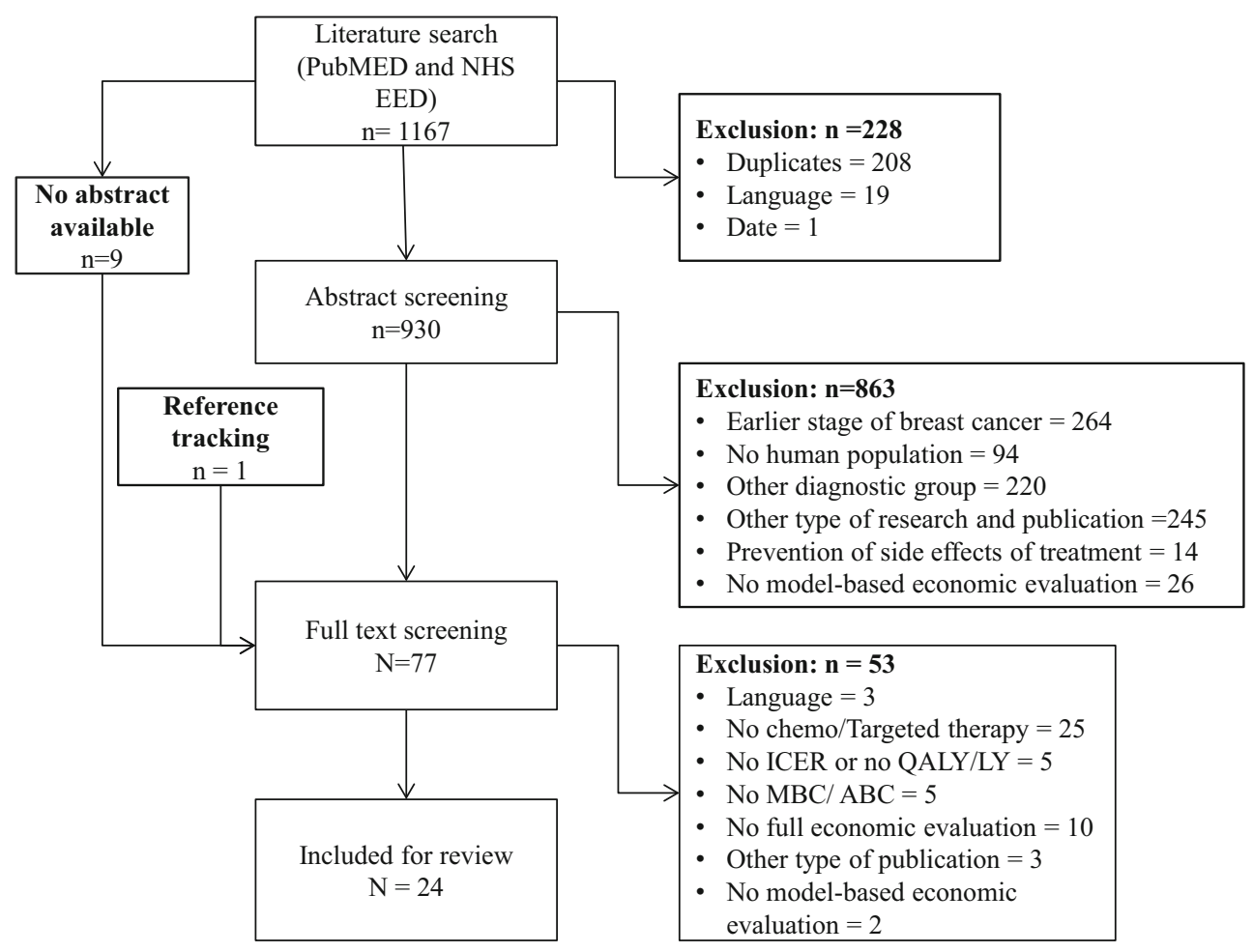

Fig. 1 Consort diagram of the selection procedure. $A B C$ advanced breast cancer, chemo chemotherapy, ICER incremental cost-effectiveness ratio, LY Life years, $M B C$ metastatic breast cancer, $Q A L Y$ Quality-adjusted life years

time horizon. Cycle time varied between one day to one and a half months (Table 1).

Extrapolation of trial data was described in nine studies. Six studies extrapolated survival data through a parametric survival model assuming a Weibull distribution, two assumed a gamma distribution and one assumed a lognormal distribution. All studies seemed to model treatment effectiveness by applying the hazard ratio of the alternative intervention to the survival function (Online Resource, Appendix 3). Lazzaro et al. was unclear about how treatment effectiveness was modelled [39]. None of the studies mentioned the use of a time-dependent treatment effect.

Health state utility values varied from 0.67 to 1.00 , from 0.61 to 0.72 and from 0.26 to 0.68 for the response, stable/ progression-free disease and progression health states, respectively. Different impacts on quality of life were associated with AEs (disutility range -0.03 to -0.25 ) (Online Resource, Appendix 4).

Three studies presented subgroup analyses: one was based on age categories [34], another on the number of chemotherapy lines received before the interventions under study [36] and the last focused on patients' body mass and surface [45].

\section{Outcomes}

Total LY and QALY ranged from 0.70 to 3.43 and from 0.29 to 2.64, respectively. Total costs ranged from $€ 1983$ to $€ 86,174$.
The NMBs ranged from $€-45,374$ to $€ 59,161 \quad(N=61)$ (Online Resource, Appendix 5). Incremental LY and QALY gained varied from 0.06 to 0.74 and from 0.05 to 0.60 , respectively. In two comparisons, the intervention dominated the comparator [36, 44], and the intervention (extendedly) dominated the comparator in six comparisons [37, 42]. For the remaining comparisons, the ICERs varied between $€ 200$ and $€ 164,000$ per LY gained $(N=24)$ and between $€ 300$ and $€ 625,000$ per QALY gained $(N=40)$. The iNMBs ranged from $€-78,574$ to $€ 15,890(N=48) ; 15(31 \%)$ of these iNMBs were positive. Norum et al. [47] results are not included in this overview because it reported a range of ICERs per LY gained instead of the results of a base-case analysis (Table 2).

\section{Quality assessment}

Most of the studies clearly described their objective $(N=16 ; 67 \%)$, comparators $(N=21 ; 88 \%)$ and model assumptions $(N=22 ; 92 \%)$. A societal perspective was used in four studies $(17 \%)$. It was unclear whether the model was appropriate for the decision problem in three studies $(N=3 ; 13 \%)$. In two of these studies, the model was not graphically represented and the possible transitions between health states were not clearly described $[39,52]$. In the third study, all health states of the model were neither mentioned nor graphically represented $(N=1 ; 4 \%)$ [41]. This hampered the authors in assessing whether the 


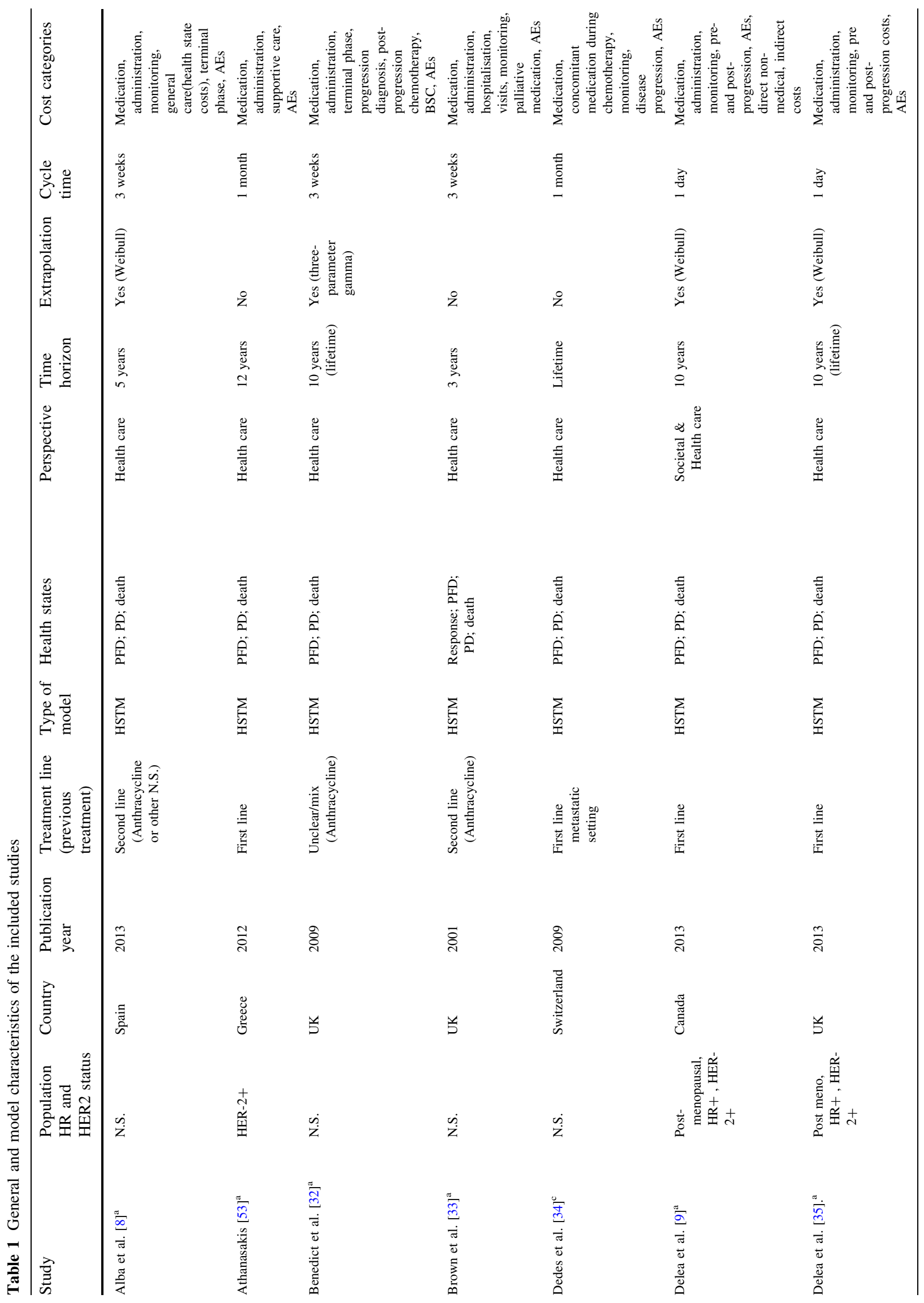




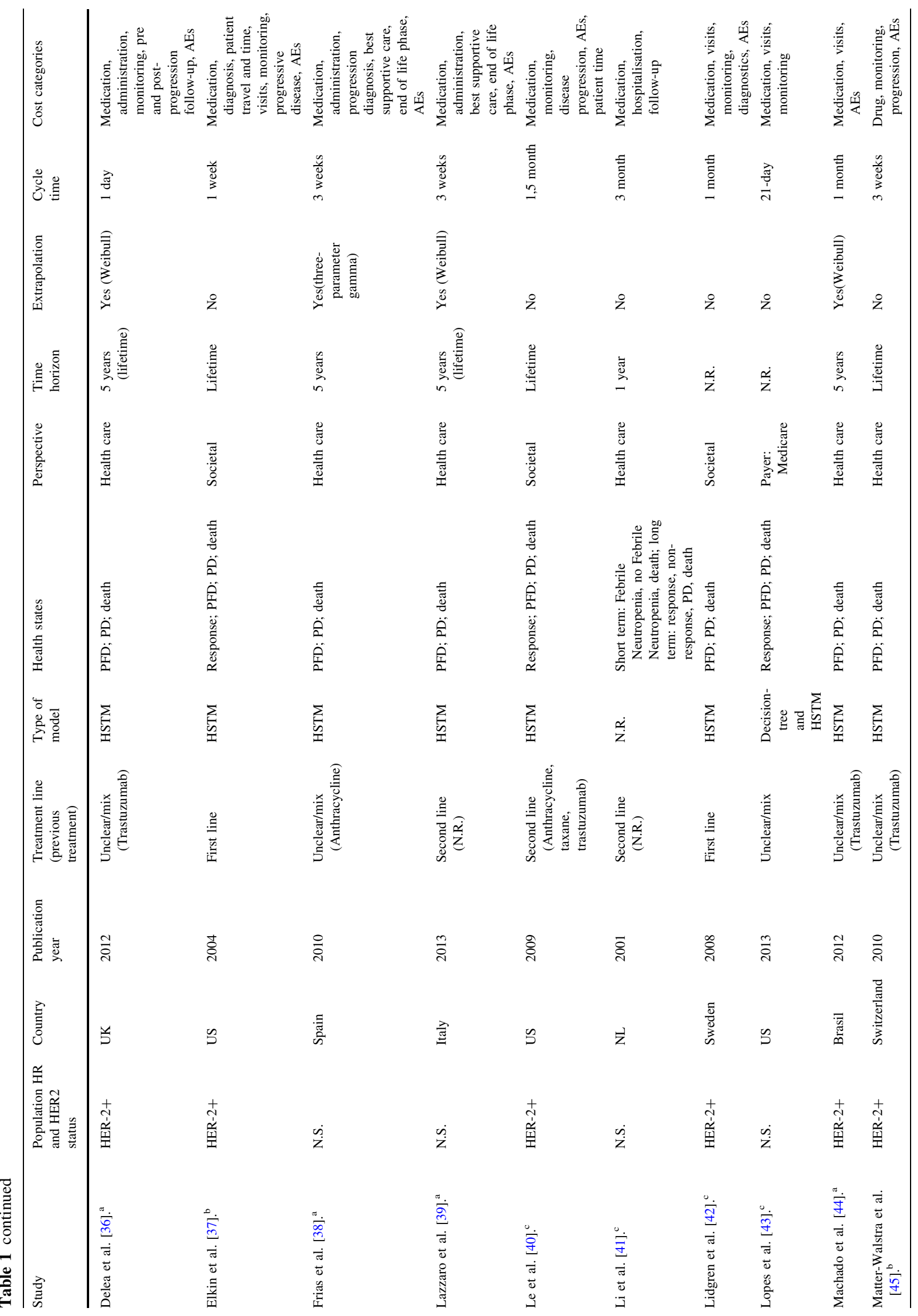




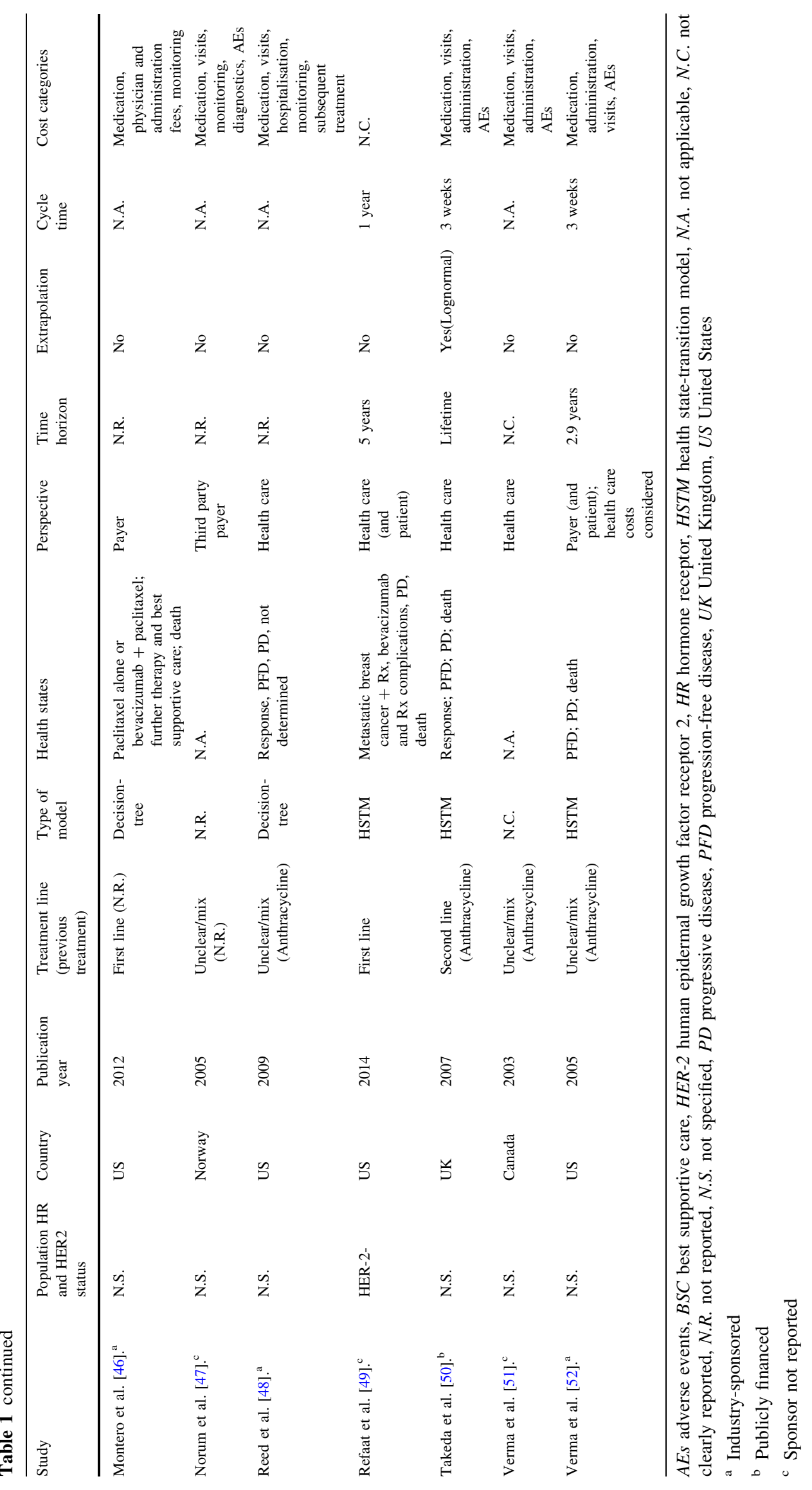




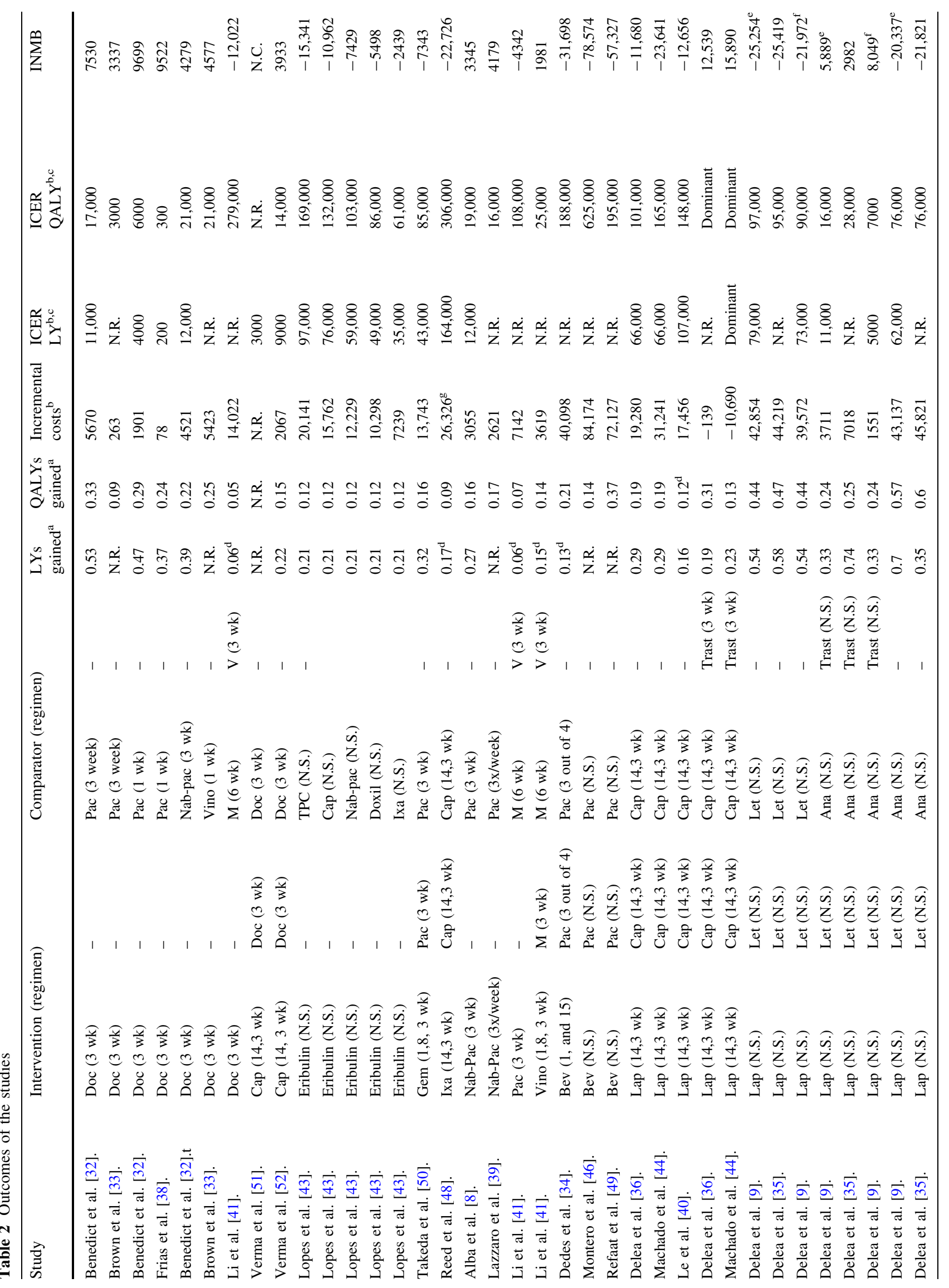




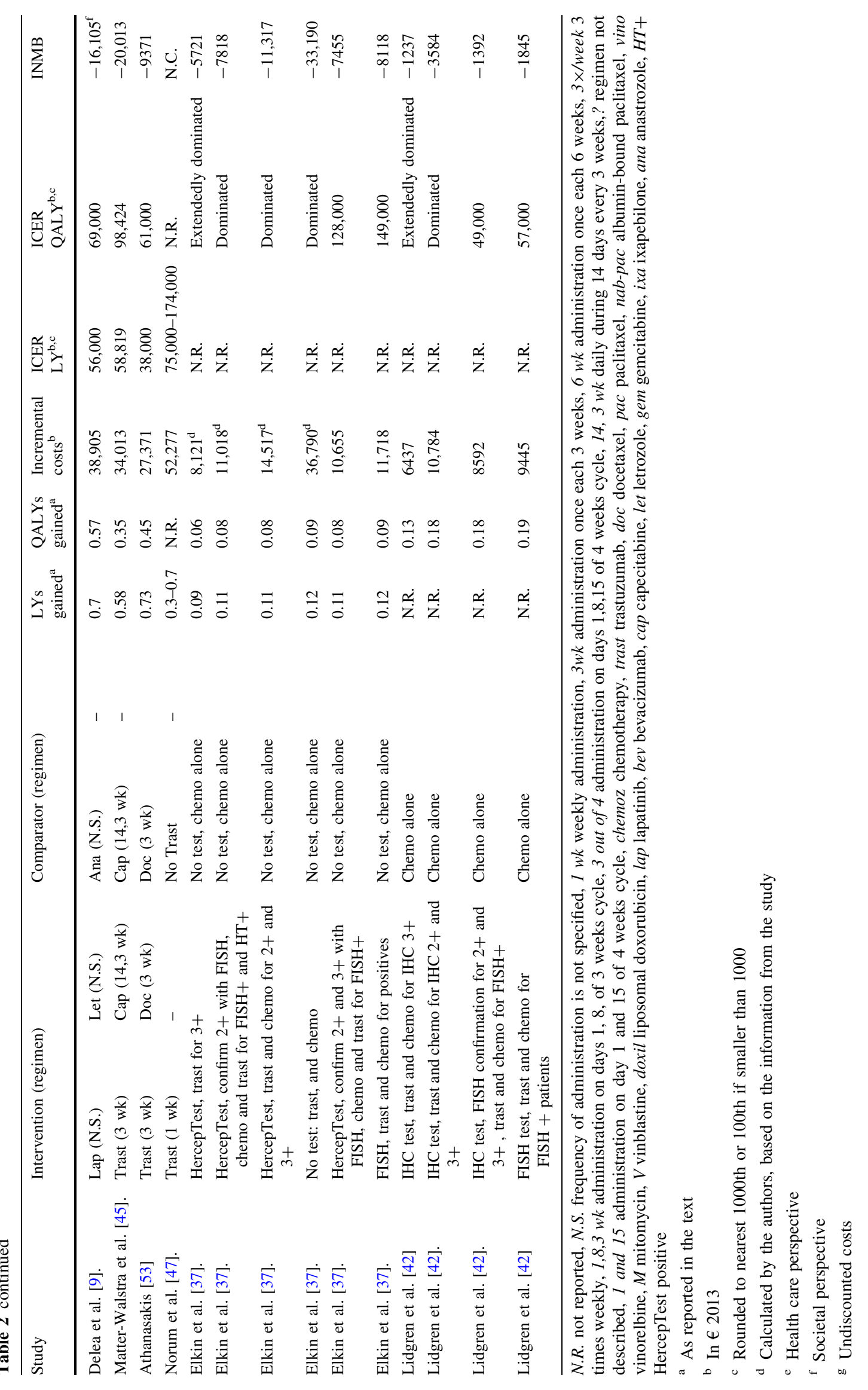


model was appropriate for the decision problem. In two studies $(8 \%)$, the model structure was not considered appropriate given the information provided. The first study did not consider costs incurred after disease progression and did not justify this choice [47]. The second study considered patients dying before treatment response assessment as 'Undetermined response'. However, patients in the 'Undetermined response' of the provided decision tree could still be subject to toxicities or progression which seemed to influence the transition probabilities of patients surviving and having an 'Undetermined response' [48]. Twenty-one $(88 \%)$ studies identified all relevant outcomes, and thirteen (54\%) clearly stated the probabilities that outcomes would happen. Outcome measurement and valuation were not clearly described in 13 studies (54\%). Thirteen (54\%) studies clearly identified all important and relevant costs, eighteen (75\%) correctly valued costs and seventeen (71\%) appropriately discounted costs. Fifteen studies (63\%) did not clearly describe how costs were measured. The authors were not able to assess the credibility and accuracy of the sources of all values in ten studies (42\%) because these were not clearly reported. Deterministic and probabilistic sensitivity analyses were performed in $23(96 \%)$ and $17(71 \%)$ studies, respectively. Ethical and distributional issues were considered in one study (4\%). None of the studies appropriately
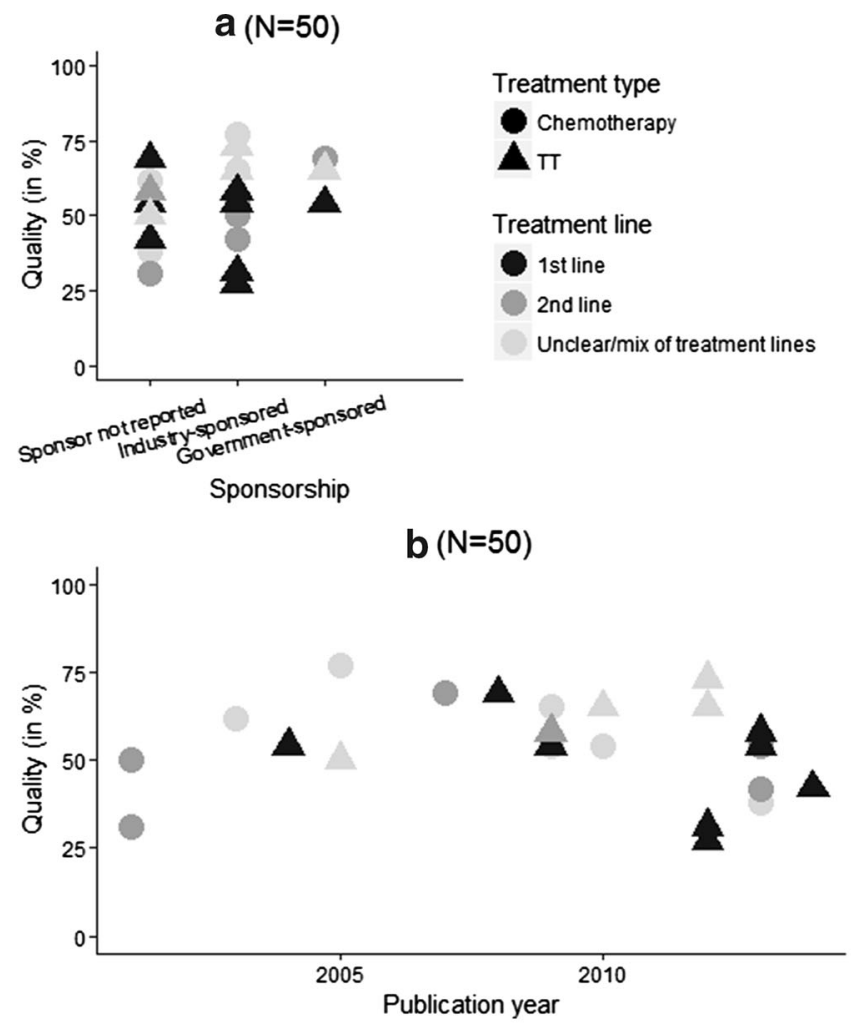

Fig. 2 Association between study quality and study characteristics and between study quality and outcomes. a Association between study quality and study sponsorship; $\mathbf{b}$ association between study quality and fulfilled all items of the quality assessment. Studies fulfilled on average 14 out of the 26 items of the checklist (range 7-20) (Online Resource, Appendix 6).

\section{Association of model characteristics with study quality and outcomes}

Study quality did not increase over time and did not seem to be associated with study sponsorship and outcomes (Fig. 2). Recently published studies more often investigated the costeffectiveness of TT which led to higher iQALY (Fig. 3). Lifetime time horizon did not seem to lead to higher health benefits (Fig. 3). Fourteen out of the twenty-five (56\%) industry-sponsored iNMBs were positive, while one of the 23 (4\%) non-industry-sponsored iNMBs (sponsorship not reported or governmental sponsorship) was positive. Finally, increased QQALY seemed to be associated with a lower iNMB (Fig. 4).

\section{Discussion}

The current literature review included 24 studies evaluating the cost-effectiveness of chemotherapy or TT for MBC treatment. Most studies (75\%) used a health state-transition
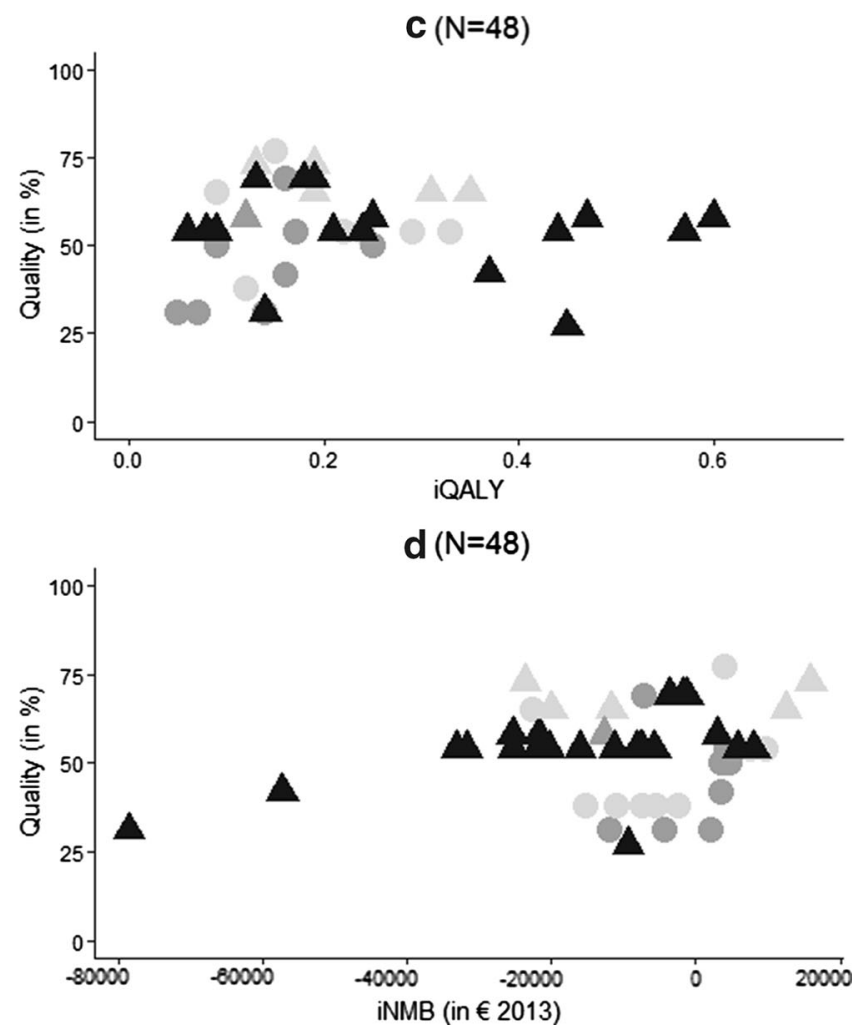

publication year; c association between study quality and iQALY; d association between study quality and iNMB; $i Q A L Y$ incremental quality-adjusted life-year; $i N M B$ incremental net monetary benefit 


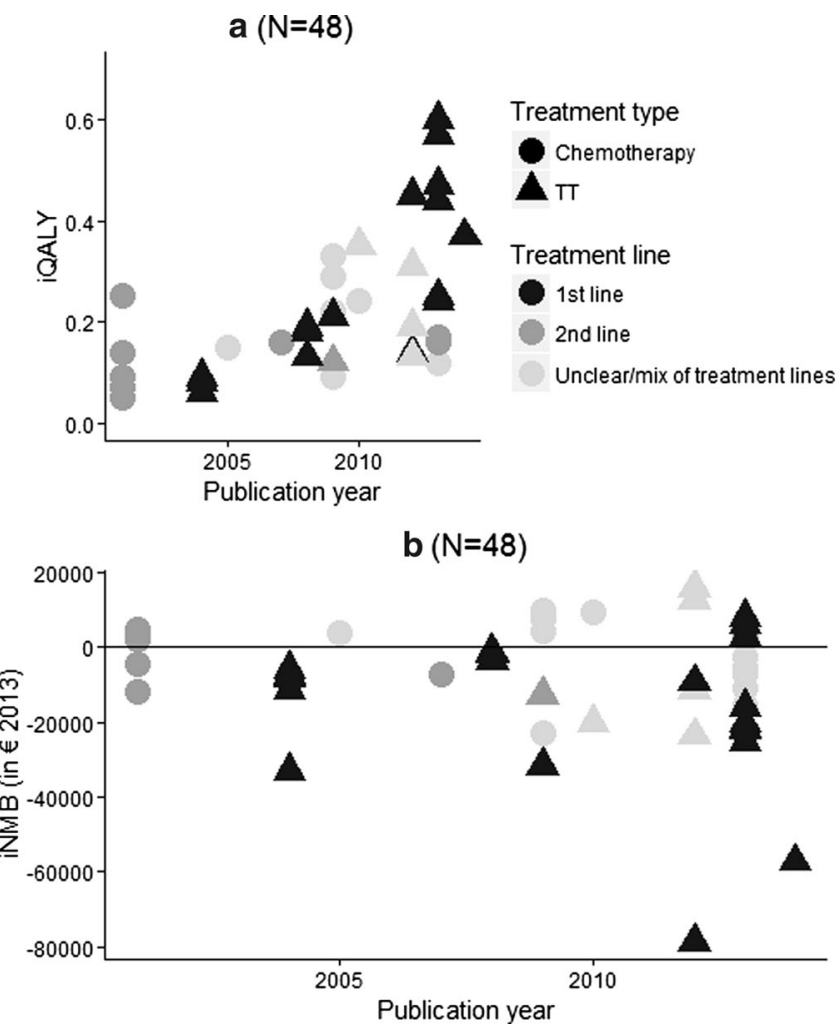

Fig. 3 Association between model characteristics and study outcomes. a Association between iQALY and publication year; $\mathbf{b}$ association between study iNMB and publication year; $\mathbf{c}$ association

approach with three health states (stable/progressionfree disease, progression and death) to model MBC, but differed with respect to time horizon, cycle times, AEs and utility values incorporated in the model. Quality of the studies was low and did not increase over time. iLY and iQALY gained ranged between 0.06 and 0.74 , and 0.05 and 0.60 , respectively. The iNMBs ranged from $€-78,574$ to $€ 15,890$ and $31 \%$ of the iNMBs were positive. TT led to higher iQALY gained. Industry-sponsored studies seemed to result in more favourable iNMB. Larger health benefits were not associated with higher value for money.

The results of the current literature study are subject to certain limitations. Firstly, the literature search was limited in time, publication type and language to make the number of included studies manageable and to retrieve up-to-date assessments potentially using state-of-the-art methodologies. Secondly, an adapted CHEC checklist, which was not specifically developed for model-based economic evaluations, was used for the quality assessment. However, this limitation is unlikely to influence our conclusions because more extensive checklists would also have identified the lack of transparency in reporting. Finally, the small number of studies investigating the same comparisons hampered comparisons of outcomes in relation to differences in model structure (e.g. number of health states) and model
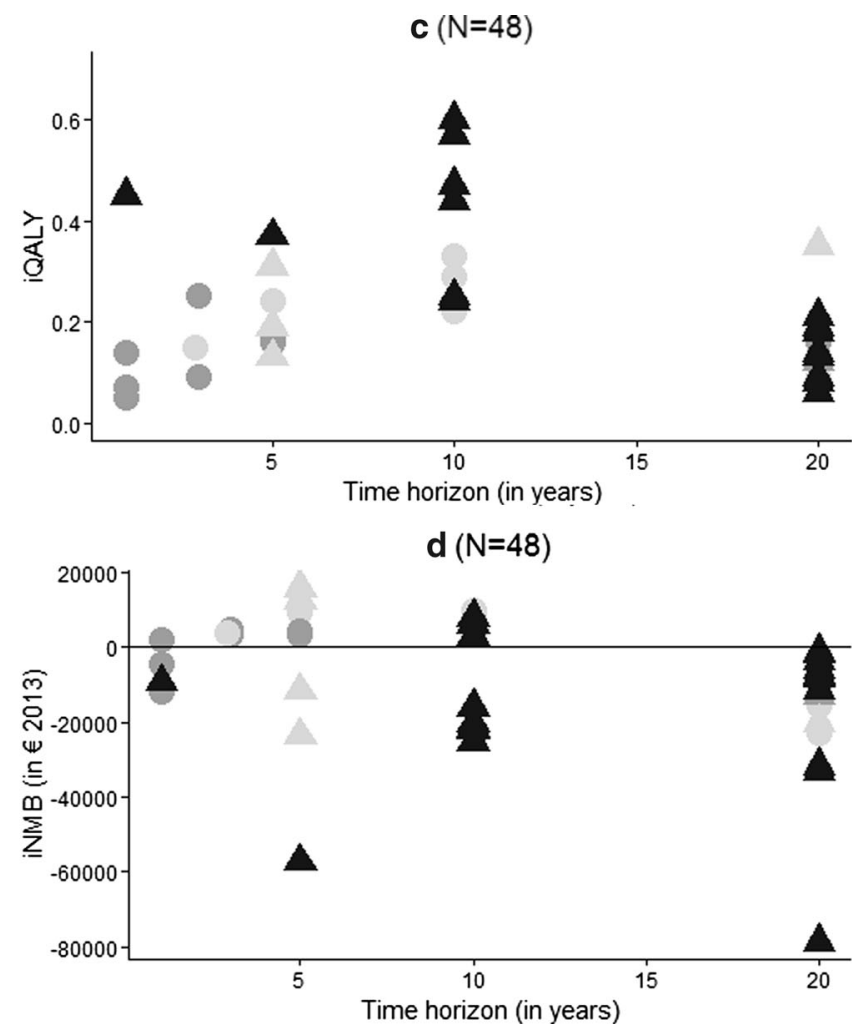

between IQALY and time horizon; $\mathbf{d}$ association between iNMB and time horizon; $i Q A L Y$ incremental quality-adjusted life-year; iNMB incremental net monetary benefit

inputs. As a result, the consistency in outcomes between different comparisons could not be investigated.

The current study did not demonstrate an association between study quality and study outcomes or sponsorship. While this lack of association is reassuring, the absence of association between study quality and time, mainly due to transparency issues, is worrisome, especially because different guidelines concerning good modelling practices and reporting have been issued [4, 54]. Transparency is a hallmark of good modelling practices because it improves the ability to interpret results and it allows to examine the validity of the models and to reproduce model outcomes [4]. Reproducibility being an essential feature of medical research, (compulsory) disclosure of all model characteristics should be encouraged.

The development of a disease-specific reference model is another solution to resolve consistency, transparency and quality issues. Disease-specific reference models would avoid duplication of work across jurisdictions and potentially accelerate coverage decision-making for $\mathrm{MBC}$ treatments. It would furthermore decrease the methodological uncertainty associated with different modelling choices made during cost-effectiveness assessments of MBC treatments. Several authors have already attempted to develop such a reference model for MBC treatments. These 
Fig. 4 Association between iQALYs and iNMBs. $i Q A L Y$ incremental quality-adjusted life-year; $i N M B$ incremental net monetary benefit

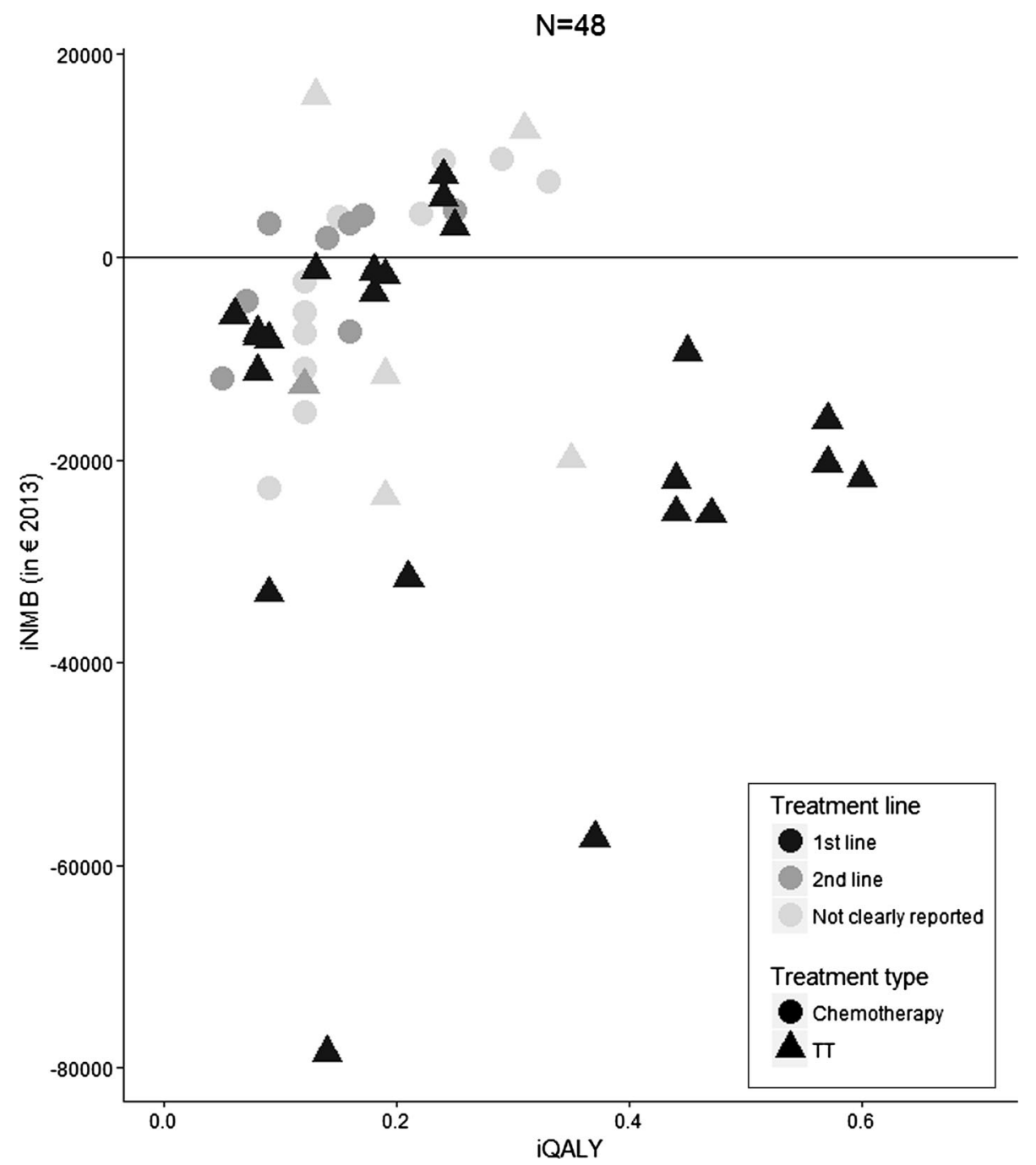

models were however limited to a specific setting or patient population [19, 20].

Increased health benefits did not lead to higher value for money, which implies that treatment costs increased when health benefits became larger. This mechanism is typical of value-based pricing frameworks. However, one might expect that prices would be set in order to remain around the willingness-to-pay threshold in a value-based pricing setting. This was not the case in the current study, i.e. $31 \%$ of the iNMBs were positive. This might indicate that valuebased pricing might be on its way in this field, but that lower prices are needed in order to meet the willingness-topay threshold. On the other hand, assessing the value of money for treatments in the metastatic setting only is misleading because using these treatments in the adjuvant setting [55] or using them more efficiently (e.g. because experience has been acquired in clinical practice) might provide better value for money. The potential value for money of these treatments over their entire life cycle may be underestimated by only assessing their value in the metastatic setting.

In conclusion, model inputs were highly variable and the quality of the included studies was low, mainly because of a lack of transparency in reporting. The development of a disease-specific reference model would increase the consistency and ensure a minimal quality of cost-effectiveness assessments for MBC treatments. Cost-effectiveness results were highly variable but, in general, MBC treatments did not provide good value for money. There was no association between study quality and study outcomes. Industrysponsored studies resulted more often in beneficial value for money of treatments compared to non-industry-sponsored studies. TT led to larger health benefits. Incremental health benefits increased over time, but were outweighed by the increased treatment costs. Consequently, increased health benefits led to lower value for money. 
Acknowledgements We would like to thank Dr. G.W.J. Frederix for his feedback on this work during LOLA HESG 2015.

\section{Compliance with ethical standards}

Conflict of interest statement XP and BR declare that they have no conflict of interest. MJ has received a grant for the set-up of a Dutch breast cancer registry from the Netherlands Organisation for Health Research and Development (ZonMw, grant number: 80-82500-989056), Roche Netherlands and Eisai.

Statement on the welfare of animals/respect of human rights This article does not contain any studies with human participants or animals performed by any of the authors.

\section{Informed consent Not applicable.}

Open Access This article is distributed under the terms of the Creative Commons Attribution 4.0 International License (http://crea tivecommons.org/licenses/by/4.0/), which permits unrestricted use, distribution, and reproduction in any medium, provided you give appropriate credit to the original author(s) and the source, provide a link to the Creative Commons license, and indicate if changes were made.

\section{References}

1. Global Cancer Observatory International Agency for Research on Cancer (World Health Organisation). http://gco.iarc.fr/today/ online-analysis-multi-bars? mode $=$ cancer $\&$ mode_population $=$ con tinents $\&$ population $=900 \&$ sex $=2 \&$ cancer $=29 \&$ type $=0 \&$ statistic $=$ $0 \&$ prevalence $=0 \&$ color_palette $=$ default. Accessed 3 Aug 2016

2. Foster TS, Miller JD, Boye ME, Blieden MB, Gidwani R, Russell MW (2011) The economic burden of metastatic breast cancer: a systematic review of literature from developed countries. Cancer Treat Rev 37(6):405-415. doi:10.1016/j.ctrv.2010.12.008

3. Drummond M, McGuire A (2001) Economic evaluation in health care: merging theory with practice. Oxford University Press, Oxford

4. Caro JJ, Briggs AH, Siebert U, Kuntz KM (2012) Modeling good research practices-overview: a report of the ISPOR-SMDM Modeling Good Research Practices Task Force-1. Value Health 15(6):796-803. doi:10.1016/j.jval.2012.06.012

5. Penaloza Ramos MC, Barton P, Jowett S, Sutton AJ (2015) A systematic review of research guidelines in decision-analytic modeling. Value Health 18(4):512-529. doi:10.1016/j.jval.2014. 12.014

6. Frederix GW, van Hasselt JG, Schellens JH, Hovels AM, Raaijmakers JA, Huitema AD et al (2014) The impact of structural uncertainty on cost-effectiveness models for adjuvant endocrine breast cancer treatments: the need for disease-specific model standardization and improved guidance. PharmacoEconomics 32(1):47-61. doi:10.1007/s40273-013-0106-x

7. Dranitsaris G, Verma S, Trudeau M (2003) Cost utility analysis of first-line hormonal therapy in advanced breast cancer: comparison of two aromatase inhibitors to tamoxifen. Am J Clin Oncol 26(3):289-296. doi:10.1097/01.coc.0000021042.55557.2b

8. Alba E, Ciruelos E, Lopez R, Lopez-Vega JM, Lluch A, Martin $M$ et al (2013) Cost-utility analysis of nanoparticle albuminbound paclitaxel versus paclitaxel in monotherapy in pretreated metastatic breast cancer in Spain. Expert Rev Pharmacoecon Outcomes Res 13(3):381-391. doi:10.1586/erp.13.18
9. Delea T, Amdahl J, Chit A, Amonkar M (2013) Cost-effectiveness of lapatinib plus letrozole in her2-positive, hormone receptor-positive metastatic breast cancer in Canada. Curr Oncol 20(5):e371

10. Le QA (2016) Structural uncertainty of markov models for advanced breast cancer: a simulation study of lapatinib. Med Decis Mak. doi:10.1177/0272989x15622643

11. Bell CM, Urbach DR, Ray JG, Bayoumi A, Rosen AB, Greenberg $D$ et al (2006) Bias in published cost effectiveness studies: systematic review. BMJ 332(7543):699-703. doi:10.1136/bmj. 38737.607558 .80

12. Greenberg D, Earle C, Fang CH, Eldar-Lissai A, Neumann PJ (2010) When is cancer care cost-effective? A systematic overview of cost-utility analyses in oncology. J Natl Cancer Inst 102(2):82-88. doi:10.1093/jnci/djp472

13. Blank PR, Dedes KJ, Szucs TD (2010) Cost effectiveness of cytotoxic and targeted therapy for metastatic breast cancer: a critical and systematic review. PharmacoEconomics 28(8):629-647. doi:10.2165/11535560-000000000-00000

14. Diaby V, Tawk R, Sanogo V, Xiao H, Montero AJ (2015) A review of systematic reviews of the cost-effectiveness of hormone therapy, chemotherapy, and targeted therapy for breast cancer. Breast Cancer Res Treat 151(1):27-40. doi:10.1007/ s10549-015-3383-6

15. Afzali HH, Karnon J, Merlin T (2013) Improving the accuracy and comparability of model-based economic evaluations of health technologies for reimbursement decisions: a methodological framework for the development of reference models. Med Decis Mak 33(3):325-332. doi:10.1177/0272989x12458160

16. Eunethta. Training material on the HTA Core Model. 2016. http://www.eunethta.eu/outputs/training-material-hta-coremodel. Accessed 21 Oct 2016

17. Frederix GW, Severens JL, Hovels AM, Raaijmakers JA, Schellens JH (2013) The cloudy crystal ball of cost-effectiveness studies. Value Health 16(6):1100-1102. doi:10.1016/j.jval.2013. 06.012

18. Eunethta. HTA Core Model ${ }^{\circledR}$ 2017. http://meka.thl.fi/htacore/ BrowseModel.aspx. Accessed 2 Feb 2017

19. Beauchemin C, Letarte N, Mathurin K, Yelle L, Lachaine J (2016) A global economic model to assess the cost-effectiveness of new treatments for advanced breast cancer in Canada. J Med Econ. doi:10.3111/13696998.2016.1151431

20. Diaby V, Ali AA, Adunlin G, Kohn C, Montero AJ (2016) Parameterization of a disease progression simulation model for sequentially treated metastatic HER2-positive breast cancer patients. Curr Med Res Opin. doi:10.1185/03007995.2016. 1149056

21. Hind D, Ward S, De Nigris E, Simpson E, Carroll C, Wyld L (2007) Hormonal therapies for early breast cancer: systematic review and economic evaluation. Health Technol Assess 11(26): $1-134$

22. Benedict A, Brown RE (2005) Review of cost-effectiveness analyses in hormonal therapies in advanced breast cancer. Expert Opin Pharmacother 6(11):1789-1801. doi:10.1517/14656566.6. 11.1789

23. Frederix GWJ, Severens JL, Hövels AM, Raaijmakers JAM, Schellens JHM (2012) Reviewing the cost-effectiveness of endocrine early breast cancer therapies: influence of differences in modeling methods on outcomes. Value Health 15(1):94-105. doi:10.1016/j.jval.2011.08.003

24. Karnon J (2006) Aromatase inhibitors in breast cancer: a review of cost considerations and cost effectiveness. PharmacoEconomics 24(3):215-232

25. Eurostat. Consumer Price Index. 2013. http://appsso.eurostat.ec. europa.eu/nui/show.do?dataset=prc_hicp_aind\&lang=en. Accessed 21 Jan 2015 
26. OECD. Consumer Price Index. 2013. http://stats.oecd.org/Index. aspx ?querytype $=v i e w \& q u e r y n a m e=221 \#$. Accessed 21 Jan 2015

27. Statcan. Consumer Price Index. 2013. http://www.statcan.gc.ca/ tables-tableaux/sum-som/101/cst01/econ46a-eng.htm. Accessed 21 Jan 2015

28. OECD. Purchase Power Parity. 2013. http://stats.oecd.org/Index. aspx? datasetcode=SNA_TABLE 4\#. Accessed 21 Jan 2015

29. Evers S, Goossens M, de Vet H, van Tulder M, Ament A (2005) Criteria list for assessment of methodological quality of economic evaluations: consensus on Health Economic Criteria. Int $\mathbf{J}$ Technol Assess Health Care 21(2):240-245

30. Soto J (2002) Health economic evaluations using decision analytic modeling. Principles and practices-utilization of a checklist to their development and appraisal. Int J Technol Assess Health Care 18(1):94-111

31. Fleeman N, Bagust A, Boland A, Dickson R, Dundar Y, Moonan $M$ et al (2011) Lapatinib and trastuzumab in combination with an aromatase inhibitor for the first-line treatment of metastatic hormone receptor-positive breast cancer which over-expresses human epidermal growth factor 2 (HER2): a systematic review and economic analysis. Health Technol Assess 15(42):1-93. doi: $10.3310 /$ hta 15420

32. Benedict A, Cameron DA, Corson H, Jones SE (2009) An economic evaluation of docetaxel and paclitaxel regimens in metastatic breast cancer in the UK. PharmacoEconomics 27(10):847-859. doi:10.2165/10899510-000000000-00000

33. Brown RE, Hutton J, Burrell A (2001) Cost effectiveness of treatment options in advanced breast cancer in the UK. PharmacoEconomics 19(11):1091-1102

34. Dedes KJ, Matter-Walstra K, Schwenkglenks M, Pestalozzi BC, Fink D, Brauchli P et al (2009) Bevacizumab in combination with paclitaxel for HER-2 negative metastatic breast cancer: an economic evaluation. Eur J Cancer 45(8):1397-1406. doi:10.1016/j. ejca.2008.12.016

35. Delea TE, Hawkes C, Amonkar MM, Lykopoulos K, Johnston SR (2013) cost-effectiveness of lapatinib plus letrozole in postmenopausal women with hormone receptor-and HER2-positive metastatic breast cancer. Breast Care 8(6):429-437. doi:10.1159/ 000357316

36. Delea TE, Tappenden P, Sofrygin O, Browning D, Amonkar MM, Karnon J et al (2012) Cost-effectiveness of lapatinib plus capecitabine in women with HER $2+$ metastatic breast cancer who have received prior therapy with trastuzumab. Eur J Health Econ 13(5):589-603. doi:10.1007/s10198-011-0323-1

37. Elkin EB, Weinstein MC, Winer EP, Kuntz KM, Schnitt SJ, Weeks JC (2004) HER-2 testing and trastuzumab therapy for metastatic breast cancer: a cost-effectiveness analysis. J Clin Oncol 22(5):854-863. doi:10.1200/jco.2004.04.158

38. Frias C, Cortes J, Segui MA, Oyaguez I, Casado MA (2010) Cost-effectiveness analyses of docetaxel versus paclitaxel once weekly in patients with metastatic breast cancer in progression following anthracycline chemotherapy, in Spain. Clin Transl Oncol 12(10):692-700. doi:10.1007/s12094-010-0579-4

39. Lazzaro C, Bordonaro R, Cognetti F, Fabi A, De Placido S, Arpino $G$ et al (2013) An Italian cost-effectiveness analysis of paclitaxel albumin (nab-paclitaxel) versus conventional paclitaxel for metastatic breast cancer patients: the COSTANza study. ClinicoEcon Outcomes Res 5:125

40. Le QA, Hay JW (2009) Cost-effectiveness analysis of lapatinib in HER-2-positive advanced breast cancer. Cancer 115(3):489-498. doi: $10.1002 / \mathrm{cncr} .24033$

41. Li N, van Agthoven M, Willemse P, Uyl-de Groot C (2001) A cost-utility analysis comparing second-line chemotherapy schemes in patients with metastatic breast cancer. Anticancer Drugs 12(6):533-540

42. Lidgren M, Wilking N, Jonsson B, Rehnberg C (2008) Costeffectiveness of HER2 testing and trastuzumab therapy for metastatic breast cancer. Acta Oncol 47(6):1018-1028. doi:10. 1080/02841860801901618

43. Lopes G, Gluck S, Avancha K, Montero AJ (2013) A cost effectiveness study of eribulin versus standard single-agent cytotoxic chemotherapy for women with previously treated metastatic breast cancer. Breast Cancer Res Treat 137(1):187-193. doi:10.1007/s10549-012-2326-8

44. Machado M, Einarson TR (2012) Lapatinib in patients with metastatic breast cancer following initial treatment with trastuzumab: an economic analysis from the Brazilian public health care perspective. Breast Cancer 4:173

45. Matter-Walstra KW, Dedes KJ, Schwenkglenks M, Brauchli P, Szucs TD, Pestalozzi BC (2010) Trastuzumab beyond progression: a cost-utility analysis. Ann Oncol 21(11):2161-2168. doi:10.1093/annonc/mdq250

46. Montero AJ, Avancha K, Gluck S, Lopes G (2012) A cost-benefit analysis of bevacizumab in combination with paclitaxel in the first-line treatment of patients with metastatic breast cancer. Breast Cancer Res Treat 132(2):747-751. doi:10.1007/s10549011-1919-y

47. Norum J, Risberg T, Olsen JA (2005) A monoclonal antibody against HER-2 (trastuzumab) for metastatic breast cancer: a model-based cost-effectiveness analysis. Ann Oncol 16(6):909-914. doi:10.1093/annonc/mdi188

48. Reed SD, Li Y, Anstrom KJ, Schulman KA (2009) Cost effectiveness of ixabepilone plus capecitabine for metastatic breast cancer progressing after anthracycline and taxane treatment. J Clin Oncol 27(13):2185-2191. doi:10.1200/jco.2008.19.6352

49. Refaat T, Choi M, Gaber G, Kiel K, Mehta M, Gradishar W et al (2014) Markov model and cost-effectiveness analysis of bevacizumab in HER2-negative metastatic breast cancer. Am J Clin Oncol 37(5):480-485. doi:10.1097/COC.0b013e31827e4e9a

50. Takeda AL, Jones J, Loveman E, Tan SC, Clegg AJ (2007) The clinical effectiveness and cost-effectiveness of gemcitabine for metastatic breast cancer: a systematic review and economic evaluation. Health Technol Assess 11(19):1-62

51. Verma S, Ilersich AL (2003) Population-based pharmacoeconomic model for adopting capecitabine/docetaxel combination treatment for anthracycline-pretreated metastatic breast cancer. Oncologist 8(3):232-240

52. Verma S, Maraninchi D, O'Shaughnessy J, Jamieson C, Jones S, Martin M et al (2005) Capecitabine plus docetaxel combination therapy. Cancer 103(12):2455-2465

53. Athanasakis K, Kyriopoulos J (2012) A cost-effectiveness analysis of trastuzumab plus docetaxel vs. docetaxel alone for the treatment of HER2-positive metastatic breast cancer in the Greek healhcare setting. Forum of. Clin Oncol 2012(3):4

54. Husereau D, Drummond M, Petrou S, Carswell C, Moher D, Greenberg D et al (2013) Consolidated health economic evaluation reporting standards (CHEERS) statement. Int $\mathrm{J}$ Technol Assess Health Care 29(2):117-122. doi:10.1017/ s0266462313000160

55. Dvortsin E, Gout-Zwart J, Eijssen EM, van Brussel J, Postma MJ (2016) Comparative cost-effectiveness of drugs in early versus late stages of cancer; review of the literature and a case study in breast cancer. PLoS ONE 11(1):e0146551. doi:10.1371/journal. pone. 0146551 